Barbosa, Paulo, Douglas Santos, Caio Lucena, Leire Ba stida, Jose Fernando Ferreira, and Gloria Cea. "The 0 CARIoT Data Acquisition App." 2020 IEEE 33rd Internat ional Symposium on Computer-Based Medical Systems (CB MS) (July 2020). doi:10.1109/cbms49503.2020.00084.

(C) IEEE

https://doi.org/10.1109/cbms49503.2020.00084 


\section{The OCARIoT Data Acquisition App}

\author{
Paulo Barbosa, Douglas Santos and \\ Caio Lucena \\ Center for Strategic Health \\ Technologies - NUTES \\ Campina Grande, Brazil \\ \{paulo.barbosa, douglas.santos, \\ caio.lucena\}@nutes.uepb.edu.br
}

\author{
Leire Bastida \\ TECNALIA, Basque Research and \\ Technology Alliance (BRTA) \\ Derio, Spain \\ leire.bastida@tecnalia.com \\ Gloria Cea \\ Life Supporting Technologies - \\ LifeSTech \\ Universidad Politécnica de Madrid \\ Madrid, Spain \\ gcea@1st.tfo.upm.es
}

\author{
José Fernando Ferreira \\ University of Fortaleza \\ Fortaleza, Brazil \\ josefernando@unifor.br
}

\begin{abstract}
We introduce the OCARIoT Data Acquisition App, a mHealth open source solution that synchronizes and manages data in Health IoT pilots. It was developed during the H2020 project entitled OCARIoT (Smart childhood Obesity CARing solution using IoT potential) and is innovative in the sense that it allows efficient IoT data collection, integration of different commercial devices, improves the user's privacy, data's security and the management of data controllers respecting legal and ethics policies. The application acts as a token manager for accessing external services. Besides the main features of the application, we report the integration of Fitbit services, and the deployment in four Health IoT pilots in Brazil, Greece and Spain. A survey was conducted with users in these three pilots and collected information about their user experience. The results presented excellent feedbacks from the users, with initial needs of synchronizing different children's accounts and privacy, which justified the development of the tool. Finally, we got feedback of improvements in ethics, technological and reuse in Health IoT pilots.
\end{abstract}

Keywords-mHealth, app, Health IoT, pilots.

\section{INTRODUCTION}

mHealth[1] includes the use of mobile and wireless devices (smart phones, tablets, smart watches, etc...) to improve the benefits for the user's health, and also better outcomes for research and services. New mHealth technologies are daily introduced in our lives through apps, platforms and new hardware mainly for consumer devices.

This paper introduces a new mobile application named OCARIoT Data Acquisition App that improves the data collection in Health IoT pilots, improving the task of data controllers in these projects. The development of such tool and the experimentation scenarios in schools is included in the scope of the OCARIoT project ${ }^{1}$. Users could be healthcare and educational professionals and managers. Moreover, the tool is fully available as open source in the github repository ${ }^{2}$.

We applied the OCARIoT Data Acquisition App with users and addressed the following research questions:

RQ1: What are the main concerns users have when collecting information in Health IoT pilots?

RQ2: What are the first observed results after using the Data Acquisition App in four Health IoT pilots?

In this sense, this paper is not only about introducing a new mHealth tool. It brings discussions and user experiences after real experimentation scenarios in four schools from three different countries. The schools were Ari de $\mathrm{Sá}^{3}$ and Daulia Bringel ${ }^{4}$ in Fortaleza-Brazil, Ellinogermaniki Agogi ${ }^{5}$ in Athens-Greece and Colegio Virgen de Europa ${ }^{6}$ in MadridSpain. Moreover, we also have the additional role of the data controller, an institution responsible for the data management of the pilots. In the case of Fortaleza-Brazil we had the University of Fortaleza playing this role, managing the data of the two local schools. In the case of Athens-Greece and Madrid-Spain, the same schools played this role.

In order to evaluate the actual feedback of users from schools and data controllers, we conducted a survey with fourteen users from the mentioned pilot scenarios. The survey had nine respondents. The contribution is to get an understanding of the improvements the OCARIoT Data Acquisition App brought for real Health IoT pilot scenarios. The survey results indicated a major concern about the efforts to synchronize all the children's wearables data and fear of exposing children's data. All the ethical and technological benefits were well recognized by users and most indicated the best prospects for the reuse of the solution in future pilots.

Moreover, the OCARIoT Data Acquisition App also addressed and solved the following problems presented by the pilots: (i) improved the agility and flexibility of data collection, since it was not necessary to login and logout to each individual child account anymore and could be easily adapted for different scenarios; (ii) improved privacy of the users, since with the token access the pilot managers can access only the necessary data for the pilot, avoiding individual aspects; and (iii) provided more visualization options, integrating data from different sensors.

\section{CONTEXT}

OCARIoT is a H2020 project composed 12 institutions from Brazil and Europe that developed a platform to promote the improvement of eating and physical disorders and also the prevention of the obesity onset for children (between 9 and 12 years old). OCARIoT also provides an IoT-based personalized coaching solution for guiding children to adopt healthy eating and physical activity behavior. The IoT network allows observing child activity patterns of daily living, health evolution, physiological and behavioral parameters, and environmental data. All this information combined with medical patterns allows OCARIoT to provide

\footnotetext{
1 ocariot.com

2 github.com/ocariot/da-app

${ }^{3}$ https://aridesa.com.br/
}

\footnotetext{
${ }^{4}$ http://www.dauliabringel.com.br/

${ }^{5} \mathrm{https}: / /$ www.ea.gr/ea/main.asp?id=100\&lag=en

${ }^{6}$ cve.edu.es
} 
a customized obesity coaching plan while enabling children to remain active and engaged in their well-being and healthy habits management. It empowers children (and also educational staff and families) in taking control of their health by collecting real-time information about nutrition and physical activity, and interconnecting healthcare professionals and children (including parents and tutors) in order to adapt the individual obesity coaching plan. OCARIoT was demonstrated and validated on three specific pilot sites in Brazil, Greece and Spain [2]. There are other interesting anti-obesity approaches based on health information extraction [3], but OCARIoT is based on prevention.

The OCARIoT platform software architecture follows the microservices paradigm, providing an API Gateway as the main entry point for initializing the microservices and their communication is managed by the Messaging Channel component, which deals with the publishing and subscription of events. The main microservices that impacts the OCARIoT Data Acquisition App are open source, as follows:

1. Account: user management and authentication. Available in github.com/ocariot/account.

2. IoT Tracking: tracking activities, sleep, environmental data and measurements. Available in github.com/ocariot/iot-tracking.

3. Data Sync: data synchronization from wearables platforms, allowing token management, revocation and to publish synchronized data in the messaging channel. Available in https://github.com/ocariot/datasync-agent.

The OCARIoT Data Acquisition App is one of the tools developed in the OCARIoT platform. Other tools are: (i) the OCARIoT App, a gamified tool that generates missions for children in order to improve their healthy habits; (ii) Embedded Gateway, a tool for collecting a managing environmental data; and (iii) the OCARIoT Dashboard, a tool visualizing and editing the main parameters of the pilot studies. In this sense, in this work we focus only on activity, sleep, although nutrition and anthropometric data are also important topics for the project and some works evolves technologies towards these topics [4]. The main users are educators, family and legal representatives, technology providers and healthcare professionals. Some functional requirement analysis initially included children also as potential user, but this possibility was discharged due to ethics and legal requirements. The main functionalities are: (i) to synchronize all the child's wearable periodically during the pilot studies; (ii) create and configure initial OCARIoT users accounts following the Data Controller policies; (iii) access some information of the children health status and integrate this information with other systems (e.g. the OCARIoT Dashboard).

In the remaining of this section we describe three scenarios of the usage of the OCARIoT Data Acquisition App for the main stakeholders. The main reported outcomes were to carry the app everywhere, to track activities and sleep data of the pilot, to see pilot's data in a nice graphical way, to have a quick and understandable way of synchronizing and managing several accounts and to feed the health model developed in the project with parameters collect through IoT.
@ Scenario1 is described in Figure 1. In this case, the children use a wearable device every day at school and at home. The educator is who manipulates and synchronizes periodically the wearable device using the OCARIoT Data Acquisition App installed in a tablet managed by the educator at school. The family just monitors the usage of the device by the children. Technology providers and healthcare professionals provide support.

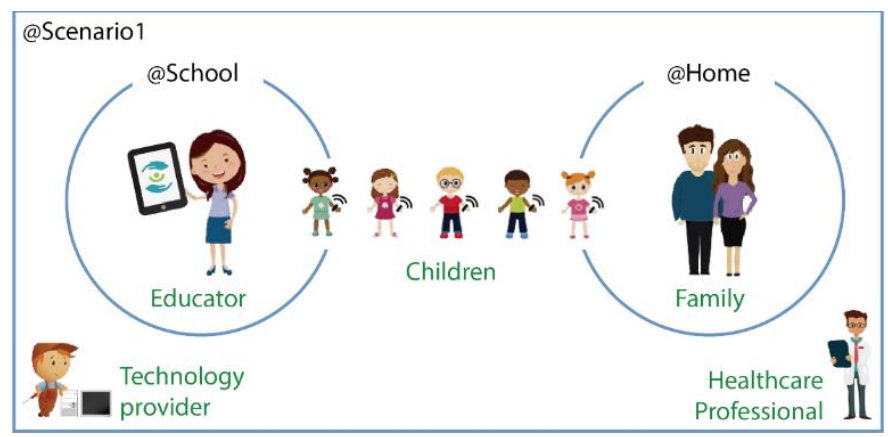

Figure 1. Scenario 1, where educators manipulate the Data Acquisition App.

@ Scenario2 is described in Figure 2. In this case, the children are who manipulates and synchronizes the wearable device. This scenario is currently suspended in the OCARIoT project due to legal and ethical aspects of the project that identified that some schools constrains the usage of smart phones by children.

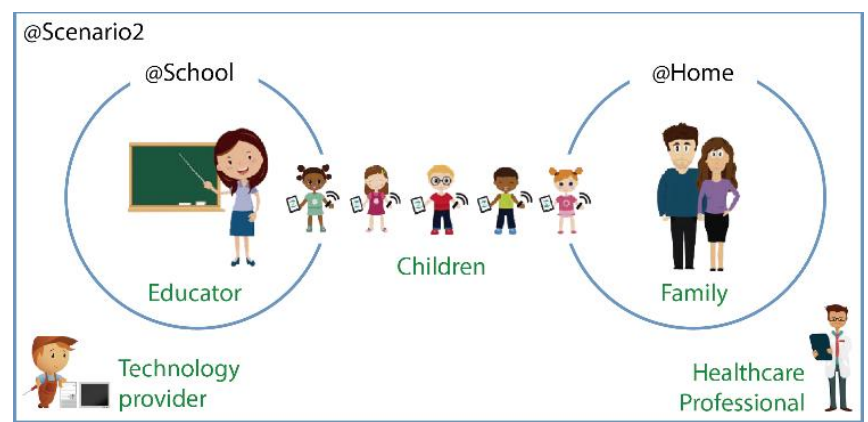

Figure 2. Scenario 2, where the children have autonomy to manipulate the Data Acquisition App.

@ Scenario3 is described in Figure 3. The family are who manipulates and synchronizes periodically the device with the OCARIoT Data Acquisition App. Technology providers and healthcare professionals provide support.

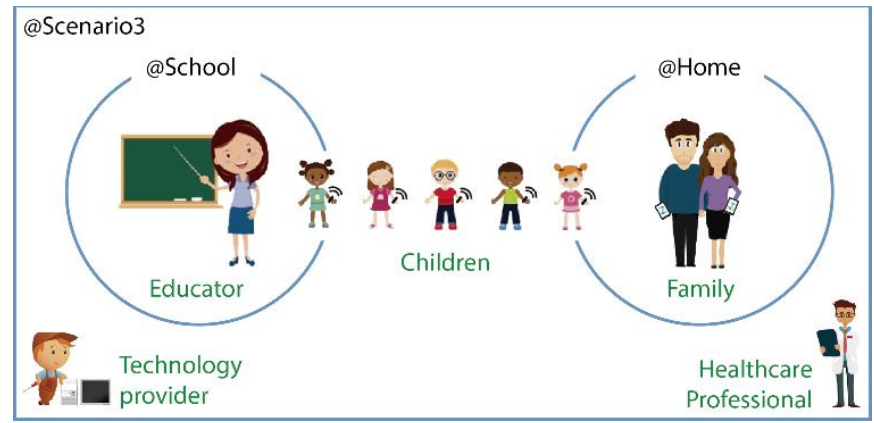

Figure 3. Scenario 3, where the family is the responsible to manipulate the Data Acquisition App. 
Due to space constraints, the focus of wearables is on the specific vendor Fitbit ${ }^{7}$, because this brand offers an interesting amount of models that we already integrate (e.g. Flex 3, Inspire, Ionic, Versa, Alta, etc...) but also because they were very open to research partnerships and opened the APIs.

The Data Acquisition App also provides features for integration of Personal Health Devices (such as weight scales, blood pressure monitors, thermometers, glucometers, cardiac monitors, etc.). In the scope of this paper, we mention the integration of Fitbit Aria, the smart weight scale from Fitbit.

\section{THE OCARIOT DATA ACQUISITION APP}

To integrate information coming from the IoT devices into the OCARIoT platform, the OCARIoT Data Acquisition App may use two different approaches, depending on the IoT device capabilities and connectivity constraints: (i) directly accessing the device through a local communication protocol as Bluetooth Low Energy; (ii) integrating with third-party platforms (e.g. Fitbit) through an open REST API and asking for permission to access the data stored on their proprietary services. In the next sections we focus on item (ii) because it is the scenario with more innovations.

\section{A. Authorization}

As OCARIoT platform accesses sleep and physical activity data from third-party platforms. Fitbit uses the OAuth 2.0, an authorization Framework that enables a third-party application to obtain limited access to an HTTP service, either on behalf of a resource owner by orchestrating an approval interaction between the resource owner and the HTTP service, or by allowing the third-party application to obtain access on its own behalf.

OAuth 2.0 defines four main $\operatorname{roles}^{8}$ : resource owner, resource server, client and authorization server. TABLE I presents a mapping between the OAuth 2.0 roles and the components playing these roles in the OCARIoT context.

TABLE I. MAPPING OAUTH 2.0 ROLES AND ENTITIES

\begin{tabular}{|l|l|}
\hline \multicolumn{1}{|c|}{ OAUTH 2.0 Role } & \multicolumn{1}{|c|}{ Entity } \\
\hline $\begin{array}{l}\text { Resource Owner: an entity capable of granting } \\
\text { access to a protected resource. When the resource } \\
\text { owner is a person, it is referred to as an end-user. }\end{array}$ & $\begin{array}{l}\text { Adult responsible } \\
\text { for child and/or } \\
\text { School. }\end{array}$ \\
\hline $\begin{array}{l}\text { Resource Server: the server hosting the protected } \\
\text { resources, capable of accepting and responding to } \\
\text { protected resource requests using access tokens. }\end{array}$ & $\begin{array}{l}\text { Wearable platform } \\
\text { (Fitbit). }\end{array}$ \\
\hline $\begin{array}{l}\text { Client: an application making protected resource } \\
\text { requests on behalf of the resource owner and with } \\
\text { its authorization. The term "client" does not imply } \\
\text { any implementation characteristics (e.g. whether } \\
\text { the application executes on a server, a desktop, or } \\
\text { other devices). }\end{array}$ & $\begin{array}{l}\text { OCARIoT Daisition App. } \\
\text { Acquata }\end{array}$ \\
\hline $\begin{array}{l}\text { Authorization Server: the server issuing access } \\
\text { tokens to the client after successfully } \\
\text { authenticating the resource owner and obtaining } \\
\text { authorization. }\end{array}$ & $\begin{array}{l}\text { Wearable platform } \\
\text { (Fitbit) }\end{array}$ \\
\hline
\end{tabular}

\section{B. Authentication}

All the interaction between the OCARIoT Data Acquisition App and the OCARIoT API is authenticated and authorized using JWT (Javascript Web Token). For this, the Data Acquisition App provides a screen to get the credentials from the children, as presented in Figure 4. It follows the OAuth 2.0 Authorization framework for accessing the physical activity and sleep data collected by wearables and stored on the wearable proprietary servers. For this, the application is registered on the Authorization Server. In this case it is the Fitbit platform, as an OAuth 2.0 client. Finally, the OCARIoT Data Acquisition App uses the Authorization Code Grant flow with the Proof Key for Code Exchange (PKCE) to mitigate against threats of interception attacks. This approach is recommended for use in native Android Apps, which is our case.
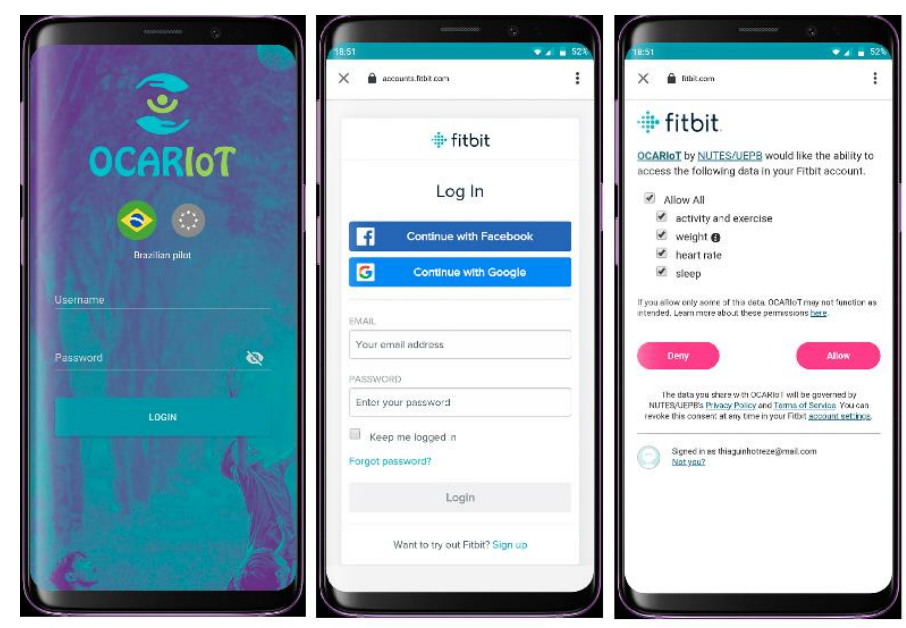

Figure 4. Main screens for authentication and Fitbit access.

Figure 4 still shows the screen for the user to allow permissions to get data from their original Fitbit account. The process presented in Figure 5 shows the flow required for the OCARIoT Data Acquisition App to obtain a valid JWT access token for use in future requests to the OCARIoT API.

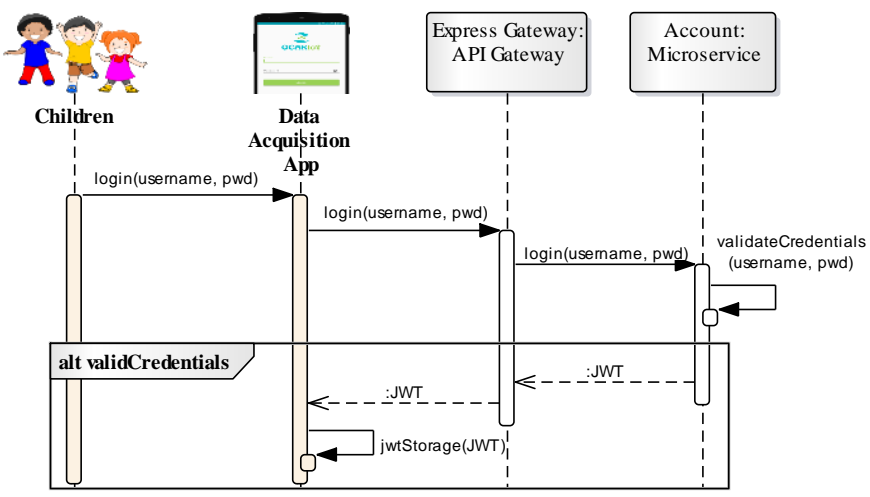

Figure 5. Authentication flow.

In this flow, a child, previously registered on the OCARIoT platform, has her credentials inserted by an adult (responsible for the child) into the OCARIoT Data Acquisition App, which exchanges them for a valid access token generated by the Account Microservice. The JWT access token contains information relevant to user's authorization and authentication such as their permissions to access the OCARIoT resources. Finally, the OCARIoT Data Acquisition App stores the JWT access token so that it can be used whenever necessary in future requests on behalf of that child. Therefore, the child just needs to provide her credentials again if she logouts of the OCARIoT Data Acquisition App or if her access token expires. 
Since the Data Acquisition App has the JWT access token, it can be used to request a resource to the OCARIoT API on behalf of the child, as illustrated in Figure 6.

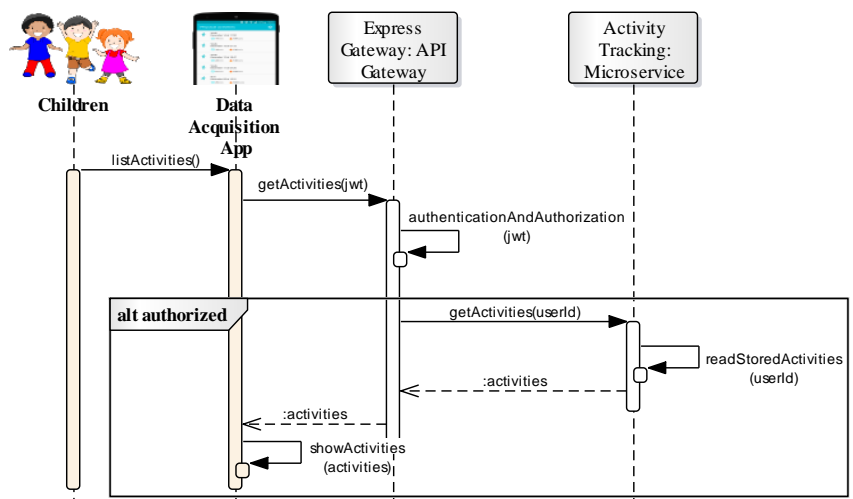

Figure 6. Example of access token usage.

In this example the adult wants to see children activity records. For this, the OCARIoT Data Acquisition App inserts the JWT access token into the OCARIoT API request. If the token is valid and contains the necessary permissions for the operation, the platform authorizes and process the request, sending back the child's activity record.

Finally, Figure 7 presents screens after synchronization. The data synchronization between Fitbit and OCARIoT takes place in the following scenarios: (i) after the adult grants access to the application to collect the Fitbit child's data; (ii) when the application is opened; and (iii) when the adult requests to see pilot's data.

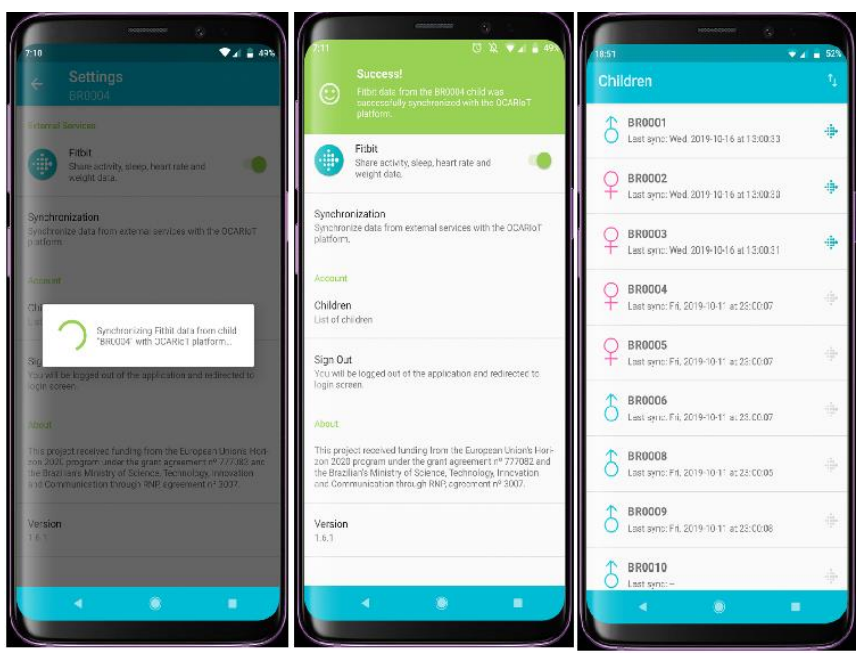

Figure 7. Fitbit successful connection.

Data synchronization is a background operation. Once data is captured from the Fitbit server, the application performs the data processing in order to convert it to the model supported by the OCARIoT platform, and synchronizes with the OCARIoT platform through the REST API.

\section{Activity and Sleep Tracking}

Fitbit provides a REST API so that third-party applications can access their user's data (with the user's consent). Its specification can be found in Fitbit website ${ }^{9}$. The OCARIoT
Data Acquisition App collects physical activities and sleep tracking using the following routes of the Fitbit REST API:

1. Fitbit activities list API command ${ }^{10}$ - Returns a list of user activities before or after a certain day;

2. Fitbit sleep list API command ${ }^{11}$ - Returns a list of user sleep records before or after a certain day.

In this sense, Figure 8 shows screens of management of activity and sleep data of users. Since the data is on the company proprietary servers, the OCARIoT can ask for access authorization to copy them to the OCARIoT servers, always without storing any personal data to identify the user. The information is displayed according to the pilot requirements.

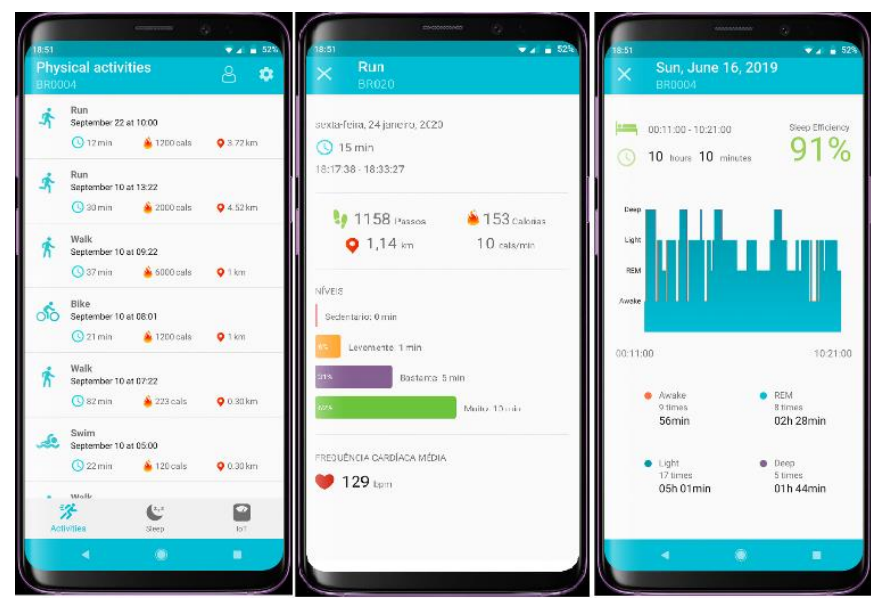

Figure 8. Screens of the activity and sleep and tracking.

\section{Data Model}

This subsection details the data synchronized from Fitbit:

1. Physical activity and its logs (steps, calories, and intensity of physical activity practices such as sedentary minutes, active minutes, etc.);

2. Sleep patterns;

3. Weight, body mass and body fat (not explored here).

TABLE II illustrates the physical activity data model. Attributes flagged with * are required. However, the activity id and user are just read-only attributes. An activity is associated with a user, being characterized as unique by the start date and child, since it is not possible for the same child to perform more than one activity simultaneously.

TABLE II. PHYSICAL ACTIVITY MODEL

\begin{tabular}{|l|l|}
\hline \multicolumn{1}{|c|}{ Name } & \multicolumn{1}{c|}{ Entity } \\
\hline id* $^{*}$ & Unique identifier of physical activity. \\
\hline name* & Name of the registered activity. \\
\hline start_time* & Date and time the physical activity was started. \\
\hline end_time* & Date and time the physical activity was finalized. \\
\hline duration* & The total duration in milliseconds of the activity. \\
\hline calories* & Total amount of calories burned during the activity. \\
\hline steps* & Total amount of steps taken during the activity. \\
\hline
\end{tabular}

${ }^{9}$ https://dev.fitbit.com/build/reference/web-api/explore/ ${ }^{10} \mathrm{https}$ ///api.fitbit.com/1/user/-/activities/list.json
${ }^{11}$ https://api.fitbit.com/1.2/user/-/sleep/list.json 


\begin{tabular}{|l|l|}
\hline \multicolumn{1}{|c|}{ Name } & \multicolumn{1}{c|}{ Entity } \\
\hline levels* & Collection containing information from activity levels in. \\
\hline child_id* & ID of Child belonging to the activity. \\
\hline heart_rate & Heart Rate measurement of physical activity. \\
\hline
\end{tabular}

TABLE III illustrates the sleep data model. A sleep record is associated with a child, being characterized as unique by the start date and time and user. It is not possible for the same child to perform more than one sleep record simultaneously.

TABLE III. SLEEP MODEL

\begin{tabular}{|l|l|}
\hline \multicolumn{1}{|c|}{ Name } & \multicolumn{1}{c|}{ Entity } \\
\hline id* & Unique identifier of the sleep record. \\
\hline start_time* & Date and time of the time the sleep log was started. \\
\hline end_time* & Date and time of the time the sleep log was finalized. \\
\hline duration* & Total duration in milliseconds of the sleep record. \\
\hline type* & Type of the Sleep Pattern (should be 'classic' or 'stages'). \\
\hline pattern* & Sleep patterns that help determine the sleep quality. \\
\hline child_id* & ID of Child belonging to sleep. \\
\hline
\end{tabular}

\section{SURVEY ABOUT THE USER EXPERIENCE IN THE PILOTS}

We conducted a survey with users focusing on a qualitative, exploratory and descriptive research. We intend to make explicit the benefits regarding the adoption of the OCARIoT Data Acquisition App starting from the main concerns users had in mind for Health IoT pilots. We had in the OCARIoT project fourteen users that effectively used and assessed the app and collected data of more than two hundred children in the Health IoT pilots until the time this paper was written. We sent the questionnaires to all the fourteen users and we got nine respondents, from Brazil, Spain and Greece.

We elaborated four very condensed and simplified questions about the main concerns when collecting data in Health IoT pilots and user's experience when managing the OCARIoT Data Acquisition App. The questions were applied as follows:

Q1. Before the Data Acquisition App, choose some concerns you had when collecting data in an Health IoT pilot:

Q2. After knowing the Data Acquisition App, could you identify ethics benefits for the Health Iot pilots?

Q3. After knowing the Data Acquisition App, could you identify some technological benefits for the Health IoT pilots?

Q4. Identify the most likely scenario for future reuse of the Data Acquisition App:

\section{A. Surverying the Data Acquistion App}

The first question, $Q 1$, asked to identify the main users concerns that the OCARIoT Data Acquisition App could solve. The answers were chosen as checkboxes, so this means that zero or multiple choices were allowed. Figure 9 shows the results with the choices of answers. The most voted item ( 8 votes) was to synchronize several accounts. Surely this is a big issue for every Health IoT project, because most of the wearables and personal health devices are designed for individual usage only. To manage all the access of users is a very consuming task. The fear of exposing children's data is another big threat, received 4 votes, and surely this is true concern for health and educational professionals because patient privacy is a major concern [5]. The lack of tools for data visualization received 3 votes, and to care about initial storage of children's data are normal operational issues and received 2 votes and, finally, no one was concerned about to learn how to manage new commercial apps for data synchronization.

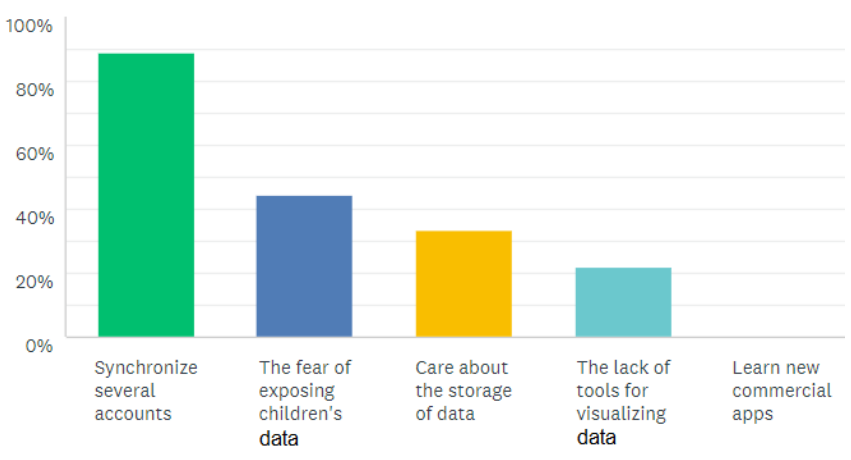

Figure 9. Answers of $Q 1$

The second question, $Q 2$, asked to identify some legal and ethics benefits. The answers were checkboxes. The results are presented in Figure 10. With 6 votes, the winner was consent tokens were managed properly and. In second place with 4 votes, we had three answers: avoided unnecessary interventions in schools, improved accountability for data controllers and avoided unnecessary interventions in schools. In this sense, we conclude that the app brings ethics benefits well recognized by the users. Dealing with legal aspects in Health IoT pilots is very big issue and tools that supports users in these issues are very welcome.

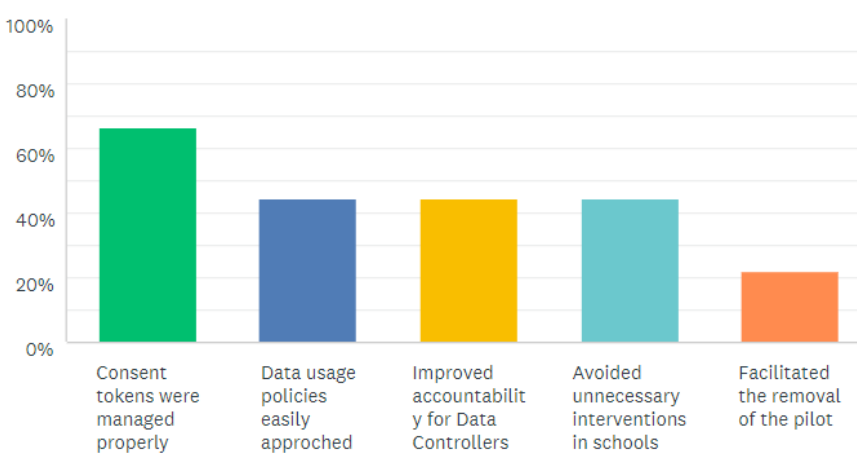

Figure 10. Answers of $Q 2$.

The third question, Q3, asked to identify some technological benefits. The answers were checkboxes. The results are presented in Figure 11. With 7 votes, we had automatic synchronization features. This feature is surely the main drive of the solution and its design was largely discussed during the OCARIoT project. However, all the other options were very recognized by users and the option with the fewest votes was all the pilot's information became centralized, which received 4 votes $(50 \%$ of the users chose also this option). Therefore, this means that the technological content of the tool was the most impressive point for the users.

Finally, the fourth question, $Q 4$, asked to identify the main scenario for future reuse of the OCARIoT Data Acquisition App. The answers were multiple choice, i.e. only one option 
could be chosen. Figure 12 shows most of the respondents identified that for medium-scale Health IoT pilots it is already necessary, with 4 votes. We also had 3 respondents choosing even for small Health IoT pilots it seemed to be necessary and 2 choosing ideal for Health IoT pilots very similar to OCARIoT. We see these results as very positive. The two worst options for possible future reuse did not receive votes.

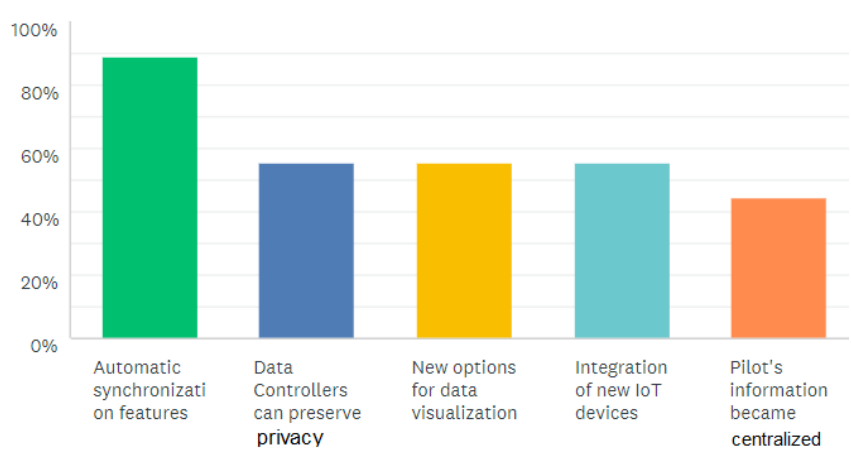

Figure 11. Answers of $Q 3$.

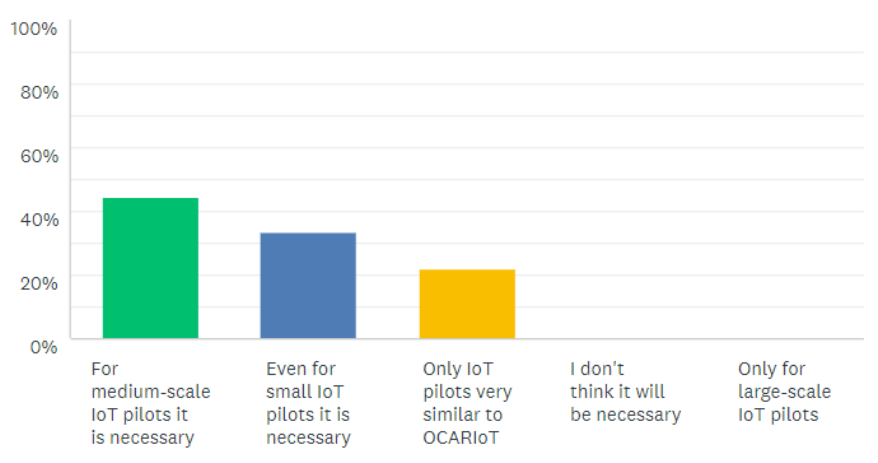

Figure 12. Answers of $Q 4$.

\section{B. Analysis and Results of the Survey}

1. The problem of having to manage the synchronization of hundreds of devices was the main initial concern of the respondent users. Surely this is a big issue for Health IoT pilots because wearables and personal health devices are designed for individual users. It was the motivation for the development of our tool.

2. The respondents were very familiar with Health IoT technologies. Evidences of that could be that no one was concerned about having to manage new commercial tools for data synchronization. Moreover, the votes recognizing the technological contributions were high and qualified.

3. The main threat to validation was the low number of respondents. Only individuals who knows effectively the app and had experiences in its usage could answer the questions. We understand the impact of the statistical significance and we are working to increase such number, but the survey was not the only contribution of the paper. Actually, these nine respondents already provided a good initial idea of the main concerns users had and the benefits they are getting using the OCARIoT Data Acquisition App. This will be important for continuous improvements in the tool.

\section{CONCLUSIONS AND FUTURE WORKS}

This paper introduced the OCARIoT Data Acquisition App. This mHealth solution provides features for synchronizing and managing data in Health IoT pilots. It takes the statement of mobile apps being used to aggregate health and fitness data [6], but advances the control over pilots. The tool was employed in four schools and received excellent feedback from the users. All the software artifacts are open source and users evaluated the tool as very likely to be reused. The main limitation of the paper is the current low number of survey respondents due to the need of familiarity with the tool.

As future works, new data collection scenarios are being negotiated, accumulating massive data in a near future.

\section{ACKNOWLEDGMENT}

The research has been performed under the OCARIoT project, funded from the European Union`s HORIZON 2020 Programme (2014-2020), ID 777082, and from the Brazilian Ministry of Science, Technology and Innovation through Rede Nacional de Ensino e Pesquisa (RNP), ID 003008. We are also thankful to developers who also contributed to the realization of the OCARIoT Data Acquisition App, namely: Jefferson Medeiros, Lucas Rocha, Aislan Monteiro, Alex Figueiredo, Thomas Fisher and Kostas Soutos.

\section{REFERENCES}

[1] P. Cipresso, S. Serino, D. Villani, C. Repetto, L. Selitti, G. Albani, A. Mauro, A. Gaggioli e G. Riva, "Is your phone so smart to affect your states? An exploratory study based on psychophysiological measures," Neurocomputing, vol. 84, 2012.

[2] L. Bastida, A. Moya, E. Gaeta, J. Filho e F. Gabler, "The Power of Gamification to Learn and Promote Healthy Habits among Children," em Proceedings of the 3rd International Symposium on Gamification and Games for Learning, Barcelona, Spain, 2019.

[3] Y. Deng, P. Dolog, J. Gass e K. Denecke, “Obesity Entity Extraction from Real Outpatient Records: When Learning-Based Methods Meet Small Imbalanced Medical Data Sets," em Proceedings of 2019 IEEE 32nd International Symposium on Computer-Based Medical Systems (CBMS), Cordoba, Spain, 2019.

[4] Z. Valero-Ramon, C. Fernandez-Llatas, A. MartinezMillana e V. Traver, "A Dynamic Behavioral Approach to Nutritional Assessment using Process Mining," em Proceedings of the 2019 IEEE 32nd International Symposium on Computer-Based Medical Systems (CBMS), Cordoba, Spain, 2019.

[5] Z. Wang, P. Myles e A. Tucker, "Generating and Evaluating Synthetic UK Primary Care Data: Preserving Data Utility \& Patient Privacy," em Proceedings of the IEEE 32nd International Symposium on ComputerBased Medical Systems (CBMS), Cordoba, Spain, 2019.

[6] V. Gay e P. Leijdekkers, "Bringing Health and Fitness Data Together for Connected Health Care: Mobile Apps as Enablers of Interoperability," J Med Internet Res, $\mathrm{n}^{\circ}$ e260, 2015. 